We do not assert that the hypertony in paraplegia is an alpha rather than a gamma type, but in about 50 injections carried out in these patients we have obtained fairly good results, especially with the triceps surae: easier mobilisation, decreased or suppressed foot clonus. Although, as a rule, the effects are never permanent and the hypertony recurs within two weeks to three months, they often give the opportunity to take further measures in rehabilitation by initiating or accelerating the patient's upright position, giving better independence and persistence of functional results. Even a transitory result will allow us to decide whether operations such as Achilles tendon lengthening or triceps surae neurotomy are indicated. The other muscles which we treated frequently with alcohol are the adductors and the biceps femoris.

We had two accidents with our patients: a painless muscle oedema, the first in a complete paraplegia, the second in an incomplete tetraplegia.

Sub-arachnoid Absolute Alcohol Injections through Vertebral Foramina. We use this technique for spasms of the hip flexor muscles. As is known, this spasticity is frequent. However, a successful treatment is difficult. It is quite a delicate technique, the aim of which is to inject the absolute alcohol through one of the vertebral foramina: $\mathrm{L}_{1}-\mathrm{L}_{2}-\mathrm{L}_{3}$, the best being $\mathrm{L}_{2}$. The patient is placed in the usual lateral position for a lumbar puncture, with the side to be treated raised, the head being in lower position. Cushions under the loin help us to put the spinal column in such a position as to obtain an angle at the point where the injection will be done. Thus, the alcohol will flow to the roots of the correspondent vertebral foramen and will denervate the muscles. We always obtained a decrease of the hip flexor spasms. We have to be very careful during the whole procedure: radiological control is necessary to know with certainty in which space the lumbar puncture needle has been introduced, constant neurological control has to be made to ensure that the injection does not reach the conus terminalis.

Amongst eight patients, we had on two occasions a slight and transient injury of the sphincter musculature. The risk of an intrathecal alcoholisation is something we must know, and the patient has to be informed of this hazard.

In conclusion, these two techniques-intramuscular and subarachnoid alcoholisations through the foramina-seem to be interesting in the treatment of spasticity in paraplegia. If there is a definite indication for such treatment these procedures have proved to be an effective and sufficient therapy with our patients and we had no serious complication with these treatments.

\title{
PHENOLISATION OF SPINAL ROOTS AND PERIPHERAL NERVES IN SPASTIC PARALYSIS
}

\author{
By M. Weiss, Stanislaw Rudnicki, Jan Haftek and Janusz Wirski \\ Clinic of Rehabilitation, Medical Academy, Warsaw, Poland
}

THE authors discuss the results of the subarachnoid phenol injection and selective peripheral nerve block with dilute phenol solution in the treatment of spasticity.

The theoretical basis of such a procedure is the differentiation of fibres which build up the spinal root and peripheral nerve in respect of their anatomical struc- 
ture. From numerous experiments it follows that the gamma fibres, which are responsible for the basic reflex arch that controls the muscle tone, are much thinner than the motor and sensory fibres.

It is common knowledge too that the nerve fibres, depending upon their thickness, have different susceptibility to damaging factors. Therefore, if the root or peripheral nerve has to be treated with an appropriate phenol solution it would result in damaging selectively the thin gamma fibres and 'the spastic arch' would be interrupted at that level.

Our own clinical material consists of 15 patients in whom unilateral or bilateral phenolisation of spinal roots was performed and of 23 patients in whom 49 peripheral nerves were phenolised. From our experience it can be concluded that in cases of complete paraplegia of the lower extremities accompanied by mass reflex synergy preventing automatic urination, subarachnoid injection of phenol solution is the method of choice. Phenol blocks of the peripheral nerves, however, are recommended in patients with spastic paresis of lower extremities without sphincter disturbance and in particular in patients with hemiparesis associated with flexion contractures of the upper extremity.

\title{
PERIPHERAL NERVE AND MOTOR POINT PHENOL BLOCKS FOR SPASTICITY
}

\author{
By H. D. Cain, A. Glass, J. Spiegler, H. Liebgold and S. Mead \\ Kaiser Foundation Rehabilitation Centre, Vallejo, California, U.S.A.
}

REDUCTION of spasticity in the extremities due to Central Nervous System diseases or disorders has been achieved by application of small amounts of 3 to 6 per cent. aqueous phenol solution to the peripheral nerve or motor points of the involved muscles. Seventy patients, ranging in age from 6 to 73, have been treated in the past $\mathrm{I} 8$ months. This simple technique is performed using an electrical stimulator which delivers a pulsating direct current through a hypodermic needle that is coated with teflon except for its tip. The peripheral nerve or motor point can first be located externally using a surface monopolar electrode. The teflon-coated needle electrode is then inserted through the skin site and visible muscle contractions occurring with minimal current intensity designates the respective peripheral nerve or motor point. From 0.3 to $0.5 \mathrm{ml}$. of aqueous phenol is injected at the motor point level or one to $10 \mathrm{ml}$. at the peripheral nerve level. Repetition of injections may be necessary for desired results. Spasticity may partially return in weeks, but satisfactory reduction for over one year has been observed. No objective or subjective sensory or motor loss has yet occurred. Mild increase in voluntary motor function has been noted in certain instances after spasticity reduction. Electromyographic studies have not revealed evidence of complete denervation after the phenol blocks. The technique is simple and effective in reducing spasticity. Film demonstration. 